\title{
New Wave Solutions for Nonlinear Differential Equations using an Extended Bernoulli Equation as a New Expansion Method
}

\author{
Serbay DURAN ${ }^{1, *}$ and Doğan KAYA \\ ${ }^{1}$ Adiyaman University, Faculty of Education, 020040 Adiyaman, Turkey \\ ${ }^{2}$ Istanbul Commerce University, Department of Mathematics, 34762 Istanbul, Turkey
}

\begin{abstract}
In this paper, we presented a new expansion method constructed by taking inspiration for the Kudryashov method. Bernoulli equation is chosen in the form of $F^{\prime}=B F^{n}-A F$ and some expansions are made on the auxiliary Bernoulli equation which is used in this method. In this auxiliary Bernoulli equation some wave solutions are obtained from the shallow water wave equation system in the general form of " $n$-order". The obtained new results are simulated by graphically in $3 \mathrm{D}$ and $2 \mathrm{D}$. To sum up, it is considered that this method can be applied to the several of nonlinear evolution equations in mathematics physics.
\end{abstract}

\section{Introduction}

Mathematical studies from nonlinear phenomena to particular case works play a significant role in applied mathematics, physics, engineering, education and so on [1]. Where at the laws of the natural and physical world are generally modeled by partial differential equations. Searching the solutions of partial differential equations is become one of the main areas of scientific works [2]. Many analytic methods have been introduced in literature. Some of them are: the Jacobi elliptic-function method [3], the sine-Gordon expansion method [4], the improved Bernoulli sub-equation function method [5] and so on.

The aim of this work, to investigate solutions of the system of the Shallow Water Wave equation with the help of the new expansion method which is a useful and popular tool for constructing exact solutions.

\section{Analysis of the Method}

\section{1 The Kudryashov Method}

This section is cover the Kudryashov method for finding wave solutions of nonlinear partial differential equations [6-7]. Accordingly, a general form of nonlinear PDE is defined as follows

\footnotetext{
* Corresponding author: sduran@adiyaman.edu.tr
} 


$$
\begin{gathered}
Q_{1}\left(\tilde{u}_{t}, \tilde{u}_{x}, \tilde{u}_{x x}, \tilde{u}_{x x x}, \ldots, x, t\right)=0, \\
\tilde{u}(x, t)=y(\xi), \quad \xi=k x-w t,
\end{gathered}
$$

where $k$ and $w$ are respectively the number of waves and the wave velocity. After (2.2) transformation is applied to the Eq. (2.1), we obtain a nonlinear ordinary differential equation for $y(\xi)$

$$
Q_{2}\left(-w y_{\xi}, k y_{\xi}, k^{2} y_{\xi \xi}, k^{3} y_{\xi \xi \xi}, \ldots\right)=0 .
$$

It is assumed that the solution $y(\xi)$ of the nonlinear equation given in Eq. (2.3) can be written in the following form:

$$
\tilde{u}(x, t)=y(\xi)=\sum_{i=0}^{M} a_{i} F^{i},
$$

where $a_{i}, \quad 1 \leq i \leq M$ are constants and $M$ is positive integers. $M$ will be determine with homogeneous balance principle. $F(\xi)$ is the following function

$$
F(\xi)=\frac{1}{1+e^{\xi}} .
$$

This function satisfies to the first order ordinary differential equation

$$
F_{\xi}=F^{2}-F \text {. }
$$

Eq. (2.6) is necessary to calculate the derivatives of function $y(\xi)$. Differentiating the expression (2.6) with respect to $\xi$ and taking into account (2.6) we have

$$
y_{\xi}=\sum_{i=1}^{M} a_{i} i(F-1) F^{i} .
$$

We substitute expressions (2.7) in Eq. (2.1). After it we take $y(\xi)$ from (2.4) into account. Thus Eq. (2.1) takes the form $P[F(\xi)]=0$ where $P[F(\xi)]$ is a polynomial of function $F(\xi)$. Then we collect all terms with the same powers of function $F(\xi)$ and equate this expression equal to zero. As a result we obtain system of algebraic equations. Solving this system we get the values of unknown parameters.

\section{2 New Expansion Method}

Instead of the auxiliary equation given in the Kudryashov method, we have specifically defined a new equation as follows.

$$
F_{\xi}=A_{1} F^{r}-A_{2} F \text { (where } F_{\xi}=\frac{d F}{d \xi}, A_{1} \text { and } A_{2} \text { are constants). }
$$

Similarly, applying steps described in the Kudryashov method (2.1), wave solutions in general form were obtained. The fundamental step of this new technique is to take full advantage of the Bernoulli equation. Auxiliary Eq. (2.8) which obtained at last step is an extended form of Eq. (2.6) in the Kudryashov method. In this new expansion method, general forms of wave solutions were obtained for special $A_{1}$ and $A_{2}$ values different from Kudryashov method. According to case of $A_{1}$ and $A_{2}$ generalized solutions of Bernoulli equation given by Eq. (2.8) are written as following:

$$
F(\xi)=(1+c \operatorname{Cosh}[(r-1) \xi]+c \operatorname{Sinh}[(r-1) \xi])^{\frac{1}{1-r}},
$$

where $A_{1}=1, A_{2}=1 ; c$ is an arbitrary constant from Eq. (2.8). 


\section{Exact Solitary Wave Solutions of the Nonlinear Differential Equations}

\subsection{Exact solitary wave solutions of the system of the shallow water wave equation}

In this example we consider the system of the shallow water wave equation [8]

$$
\begin{gathered}
\tilde{u}_{t}+\tilde{u}_{x} \tilde{v}+\tilde{v}_{x} \tilde{u}+\tilde{v}_{x x x}=0 \\
\tilde{v}_{t}+\tilde{u}_{x}+\tilde{v} \tilde{v}_{x}=0 . \\
\tilde{u}(x, t)=\tilde{u}(\xi) \text { and } \tilde{v}(x, t)=\tilde{v}(\xi), \quad \xi=k x+w t .
\end{gathered}
$$

Using Eq. (3.2) on Eq. (3.1) and integrating with respect to $\xi$ yields, we yield following equation,

$$
\begin{aligned}
& w \tilde{u}+k \tilde{u} \tilde{v}+k^{3} \tilde{v}^{\prime \prime}=0, \\
& w \tilde{v}+k \tilde{u}+\frac{k}{2} \tilde{v}^{2}=0 .
\end{aligned}
$$

The solutions of the systems, we are looking for in stated in the form

$$
\tilde{u}(x, t)=\tilde{u}(\xi)=\sum_{i=0}^{M_{1}} a_{i} F^{i}, \quad \tilde{v}(x, t)=\tilde{v}(\xi)=\sum_{i=0}^{M_{2}} b_{i} F^{i} .
$$

Balancing the highest linear terms with the highest nonlinear terms Eq. (3.3) we can found $M_{1}=2 r-2, M_{2}=r-1$ and

$$
\tilde{u}=a_{0}+a_{1} F+a_{2} F^{2}+\ldots+a_{2 r-2} F^{2 r-2}, \quad \tilde{v}=b_{0}+b_{1} F+\ldots+b_{r-1} F^{r-1}
$$

Substituting (3.5) into Eq. (3.3) yields a set of algebraic equations for $k, w, a_{0}, a_{1}, \ldots, a_{2 r-2}, b_{0}, b_{1}, \ldots, b_{r-1}$. With the aid of Mathematica from the solutions of the system, we can found

\section{Case I:}

$$
\begin{gathered}
k \neq 0, \quad w \neq 0, \quad m \in(0, r-1), \quad l \in(r-1,2 r-2), \text { where } m, l \in I N^{+}, \\
a_{r-1}=(2 r-2) A_{2} w, \quad a_{2 r-2}=-\frac{(2 r-2) A_{2}^{2} w}{A_{1}}, \quad b_{0}= \pm 2 \sqrt{(r-1) A_{1} w}, \\
b_{n-1}=\mp \frac{2 A_{2} \sqrt{(r-1) A_{1} w}}{A_{1}}, \quad k=\mp \frac{\sqrt{w}}{\sqrt{(r-1) A_{1}}}, \\
a_{0}=a_{m}=a_{l}=b_{m}=0, \quad A_{1} \neq 0, \quad \alpha \neq 0, \quad r>2 .
\end{gathered}
$$

\section{Case II:}

$k \neq 0, \quad w \neq 0, \quad m \in(0, r-1), \quad l \in(r-1,2 r-2), \quad$ where $m, l \in I N^{+}$,

$$
\begin{aligned}
& a_{r-1}=-(2 r-2) A_{2} w, \quad a_{2 r-2}=\frac{(2 r-2) A_{2}^{2} w}{A_{1}}, \quad b_{0}= \pm 2 i \sqrt{(r-1) A_{1} w}, \\
& b_{r-1}=\mp \frac{2 i A_{2} \sqrt{(r-1) w}}{\sqrt{A_{1}}}, \quad k= \pm \frac{i \sqrt{w}}{\sqrt{(r-1) A_{1}}}, \\
& a_{0}=a_{m}=a_{l}=b_{m}=0, \quad A_{1} \neq 0, \quad \alpha \neq 0, \quad i^{2}=-1, \quad r>2 .
\end{aligned}
$$

\section{Solution 1:}

We obtain the solution of Eq. (3.1) for case 1 as following 


$$
\begin{aligned}
\tilde{u}(x, t) & =(2 r-2) A_{2} w\left(\left(\frac{A_{2}}{A_{1}}+c \operatorname{Cosh}\left[A_{1}(r-1) \xi\right]+c \operatorname{Sinh}\left[A_{1}(r-1) \xi\right]\right)^{\frac{1}{1-r}}\right)^{r-1}- \\
& =-\frac{(2 r-2) A_{2}^{2} w}{A_{1}}\left(\left(\frac{A_{2}}{A_{1}}+c \operatorname{Cosh}\left[A_{1}(r-1) \xi\right]+c \operatorname{Sinh}\left[A_{1}(r-1) \xi\right]\right)^{\frac{1}{1-r}}\right)^{2 r-2}, \\
\tilde{v}(x, t) & = \pm 2 \sqrt{(r-1) A_{1} w} \mp \frac{2 A_{2} \sqrt{(r-1) w}}{\sqrt{A_{1}}}\left(\left(\frac{A_{2}}{A_{1}}+c \operatorname{Cosh}\left[A_{1}(r-1) \xi\right]+c \operatorname{Sinh}\left[A_{1}(r-1) \xi\right]\right)^{\frac{1}{1-r}}\right)^{r-1}, \\
\xi & = \pm \frac{\sqrt{w}}{\sqrt{(r-1) A_{1}}} x+w t .
\end{aligned}
$$

\section{Solution 2:}

We obtain the solution of Eq. (3.1) for case 2 as following

$$
\begin{aligned}
\tilde{u}(x, t) & =-(2 r-2) A_{2} w\left(\left(\frac{A_{2}}{A_{1}}+c \operatorname{Cosh}\left[A_{1}(r-1) \xi\right]+c \operatorname{Sinh}\left[A_{1}(r-1) \xi\right]\right)^{\frac{1}{1-r}}\right)^{r-1}+ \\
& =+\frac{(2 r-2) A_{2}^{2} w}{A_{1}}\left(\left(\frac{A_{2}}{A_{1}}+c \operatorname{Cosh}\left[A_{1}(r-1) \xi\right]+c \operatorname{Sinh}\left[A_{1}(r-1) \xi\right]\right)^{\frac{1}{1-r}}\right)^{2 r-2}, \\
\tilde{v}(x, t) & = \pm 2 i \sqrt{(r-1) A_{1} w} \mp \frac{2 i A_{2} \sqrt{(r-1) w}}{\sqrt{A_{1}}}\left(\left(\frac{A_{2}}{A_{1}}+c \operatorname{Cosh}\left[A_{1}(r-1) \xi\right]+c \operatorname{Sinh}\left[A_{1}(r-1) \xi\right]\right)^{\frac{1}{1-r}}\right)^{r-1}, \\
\xi & = \pm \frac{i \sqrt{w}}{\sqrt{(r-1) A_{1}}} x+w t .
\end{aligned}
$$

Specifically, in the solution (3.8) we obtained solution below for $n=2, A=1$ and $B=1$.

$$
\begin{aligned}
& \tilde{u}(x, t)=\mp \frac{2 c w(\operatorname{Cosh}[t w \pm \sqrt{w} x]+\operatorname{Sinh}[t w \pm \sqrt{w} x])}{(1+c \operatorname{Cosh}[t w \pm \sqrt{w} x]+c \operatorname{Sinh}[t w \pm \sqrt{w} x])^{2}}, \\
& \tilde{v}(x, t)=\mp 2 \sqrt{w}\left(1-\frac{1}{1+c \operatorname{Cosh}[t w \pm \sqrt{w} x]+c \operatorname{Sinh}[t w \pm \sqrt{w} x]}\right)
\end{aligned}
$$
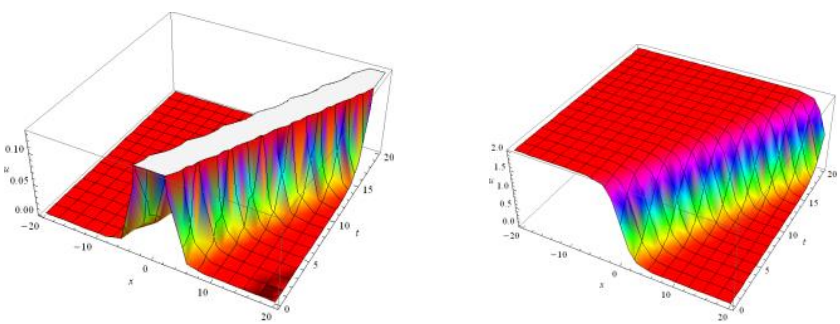

Fig. 1. $\tilde{u}(x, t)$ and $\tilde{v}(x, t)$ three dimensional wave view, by substituting the values $\alpha=1, w=1, c=1$ respectively for solution (3.10) of the Eq. (3.1). 


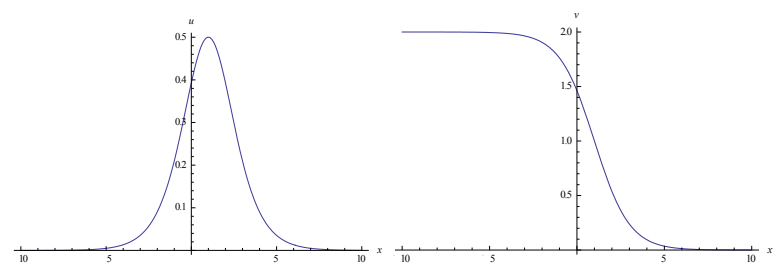

Fig. 2. $\tilde{u}(x, t)$ and $\tilde{v}(x, t)$ two dimensional wave view, by substituting the values

$\alpha=1, w=1, c=1$ and $t=1$ respectively for solution (3.10) of the Eq. (3.1).

\section{Conclusions}

In this study, the new expansion method is given by using auxiliary equation. Solutions different from literature and more general solutions have been achieved by applying this method, these solutions have been illustrated by 2 and 3 dimensional graphics. The expansion method we have devised may be used to create solutions to other nonlinear equations and nonlinear system of equations.

\section{References}

1. E. Yuce, A. Ö. Tarakçioglu, Attitudes of Computer Engineering Department Students towards ESP Courses Integrated to Foreign Language Courses: Tunceli University Case, Bilim ve Gençlik Dergisi, 1(1), 47-57, (2013).

2. A. Yokus, H. M. Baskonus, T. A. Sulaiman \& H. Bulut, Numerical simulation and solutions of the two-component second order KdV evolutionary system. Numerical Methods for Partial Differential Equations, 34(1), 211-227, (2018).

3. L. Shikuo, Jacobi elliptic function expansion method and periodic wave solutions of nonlinear wave equations, Physics Letters A 289.1-2, 69-74, (2001).

4. A. Yokus, T. A. Sulaiman, H. Bulut, On the analytical and numerical solutions of the Benjamin-Bona-Mahony equation. Opt. Quant. Electron. 50, 31, (2018).

5. H. M. Baskonus and H. Bulut, Exponential Prototype Structure for (2+1)-Dimensional Boiti-Leon-Pempinelli systems in Mathematical Physics, Waves in Random and Complex Media, 26(2), 189-196, (2016).

6. N. A. Kudryashov, On Types of Nonlinear Nonintegrable Equations with Exact Solutions, Physics Letters A, 155, 269-275, (1991).

7. N. A. Kudryashov, One method for finding exact solutions of nonlinear differential equations, Communications in Nonlinear Science and Numerical Simulation 17.6, 2248-2253, (2012).

8. S. Duran, and D. Kaya, Applications of a New Expansion Method for Finding Wave Solutions of Nonlinear Differential Equations, World Applied Sciences Journal 18.11, 1582-1592, (2012). 